\title{
基于脉冲信号概念的往复压缩机流量调节方法分析
}

\author{
顾兆林 ${ }^{\left(1^{*}\right.}$ ，侯雄坡 ${ }^{(2 *}$ ，王赞社 ${ }^{(1)}$ ，冯诗愚 ${ }^{(3)}$ ，高秀峰 ${ }^{(2)}$ ，李云 ${ }^{(2)}$ \\ (1) 西安交通大学人居环境与建筑工程学院, 西安 710049; \\ (2) 西安交通大学能源与动力工程学院, 西安 710049; \\ (3) 南京航空航天大学航空宇航学院, 南京 210016 \\ * 联系人, E-mail: guzhaoln@mail.xjtu.edu.cn; houxp@ mail.xjtu.edu.cn
}

2010-12-13 收稿, 2011-04-15 接受

国家自然科学基金资助项目(51006077)

\begin{abstract}
摘要 大型往复压缩机是流程行业中应用广泛、能耗巨大的关键设备, 压缩机在运行中经常需要 进行流量调节, 通过先进的流量调节技术达到压缩机的节能运行是流程行业实现节能减排的有 效途径. 首先分析了压缩机流量调节方法的理论依据和发展现状, 将压缩机工作过程与脉冲信 号进行了概念性类比, 从脉冲信号处理的角度将流量调节技术重新分类为调频、调幅等类型, 并 对较为重要的变余隙调节、部分行程压开吸气阀和全行程压开吸气阀等调节方法的原理和适用 性进行了述评. 以脉冲信号概念为工具, 介绍了占空比调制的压缩机流量调节方法, 详细分析了 新方法与已有吸气阀调节方法在原理上的异同, 并比较了各自的调节效果和实现方法. 对比结 果表明, 占空比调制模式在流量调节的精度、压力平稳性、节能效果和可靠性等方面具有优良的 综合性能. 各种压开吸气蔺调节方法使气体发生回流, 都会不同程度的出现呼吸效应. 分析了呼 吸效应对流量调节性能和安全的影响, 需要对呼吸效应加以重视.
\end{abstract}

关键词

往复压缩机

流量调节

压开吸气沎

占空比调制
大型往复压缩机 (以下简称压缩机) 具有供给压 力范围宽、变工况范围宽和效率高等优点, 一直是石 化、化工等流程行业中的关键设备. 一套 $10 \mathrm{Mt} / \mathrm{a}$ 炼油 装置需要压缩机组 10 15 台, 一套 $1 \mathrm{Mt} / \mathrm{a}$ 乙烯装置需 要压缩机组 20 30 台, 功率大都在 $2000 \mathrm{~kW}$ 甚至 $3000 \mathrm{~kW}$ 以上, 工艺压缩机的节能运行对于国民经济 整体节能减排的意义越来越重要. 压缩机一般是按 规定的工艺条件和额定的流量进行设计的, 在选型 时主要考虑对峰值流量的满足, 这使得压缩机运行 时流量经常处于富余状态, 有效负荷率常常只有 60\% 85\%; 另一方面, 流程的原料性质、生产工艺及 产品结构等经常发生变化, 对流量的需求也相应的 改变, 必须提供合适的流量调控手段. 压缩机 的功 率与流量一般是成正相关关系, 压缩机实现高效、精 确的气量调节是整个流程行业实现大规模节能的关 键技术和有效途径. 对工艺压缩机而言, 流量调
节机构应保证高度的可靠性, 实施调节的过程中要 保证供气的连续和压力的稳定. 对压缩机流量调节 的要求可以概括为: 可靠、稳定、精确、节能.

\section{1 压缩机流量调节的基本理论}

\section{1 压缩机流量调节的热力学原理}

往复压缩机将气体密闭在一个气缸中, 通过活 塞的移动缩小该容积、使气体增压. 如图 1 中虚线连 成的封闭曲线，压缩机依次经过吸气(d-f-a)、压缩 (a-b)、排气(b-c)和膨胀 (c-d) 等 4 个连续的热力过程而 构成一个工作循环. 其中膨胀过程的出现是由于排 气终了时在气缸余隙容积 $V_{0}$ (对应 $\mathrm{c}$ 点)内还存在着未 排净的高压气体.

由于上述工作原理，压缩机是间歇吸气、间歇排 气的, 因此所谓压缩机的流量实际是指一段时间的 


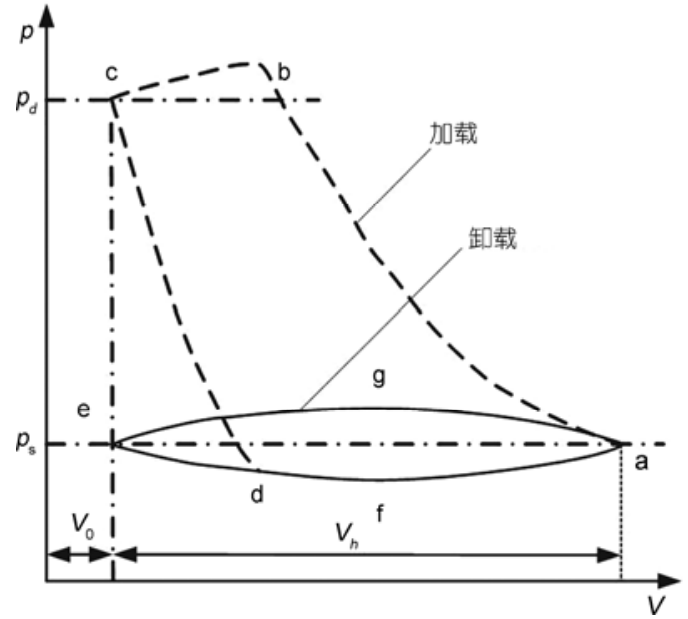

图 1 压缩机实际工作循环指示图

平均流量, 其定义为: 在规定的进口状态和排气压力 等条件下，单位时间内进人压缩机气缸的气体换算 到标准状况下 $\left(101325 \mathrm{~Pa}\right.$ 和 $\left.0^{\circ} \mathrm{C}\right)$ 的体积流量, 也可表 示为质量流量. 当气体被视作理想气体时, 压缩机 1 级缸的质量流量可表示为 ${ }^{[1]}$

$$
Q_{m}=\frac{V_{s 1} p_{s 1}}{R_{g} T_{s 1}} \cdot \frac{n}{60}=\lambda_{v 1} \lambda_{p 1} \lambda_{T 1} \lambda_{11} \frac{n V_{h 1} p_{s 1}}{60 R_{g} T_{s 1}},
$$

其中, 下标 1 表示 1 级缸, $n$ 为转速, $V_{h}, p_{s}, T_{s}, R_{g}$ 分别 为气缸的行程容积、吸气压力、吸气温度和气体常数. 根据经验 ${ }^{[1]}$, 压力系数 $\lambda_{p 1}=0.95 \sim 0.98$, 温度系数 $\lambda_{T 1}=0.93 \sim 0.97$, 泄漏系数 $\lambda_{l 1}=0.95 \sim 0.97$, 而容积系数 $\lambda_{v 1}$ 由下式确定：

$$
\lambda_{v 1}=1-\alpha_{1}\left(\varepsilon_{1}^{\frac{1}{m}}-1\right),
$$

其中, $\alpha$ 为相对余隙容积系数, $\alpha=V_{0} / V_{h}, \varepsilon$ 为名义压比, $m$ 为膨胀过程指数.

从热力学角度分析, 可以通过调节式(1)中各参 数的值而实现压缩机的流量调节. 被调参数的选取, 除了可行性以外, 更多的是从经济性即压缩机功率 的角度进行衡量. 压缩机的功率是各级指示功率与 机械损失之和, 其中机械损失可以通过压缩机的机 械效率包含进去. 正常工作时, 第 $j$ 级气缸的指示功 率 $P_{i j}$ 按下式 ${ }^{[1]}$ 计算: $\left.P_{i j}=\frac{n}{60}\left(1-\delta_{s j}\right) p_{s j} \lambda_{v j} V_{h j} \frac{m_{j}}{m_{j}-1}\left\{\varepsilon_{j}\left(1+\delta_{s j}+\delta_{d j}\right)\right]^{\frac{m_{j}-1}{m_{j}}}-1\right\}$,

其中, $\delta_{s}, \delta_{d}$ 分别为吸气压力损失和排气压力损失.

压缩机的比功率定义为压缩单位质量(或标况下
单位体积)的气体所消耗的功. 这一概念常用于比较 空气压缩机的性能. 当流程压缩机进行流量调节时, 一般都会影响到压缩机的功率. 先进的调节方法应 使得实际功率随流量的降低而成比例降低, 即应保 持比功率不变. 本文将这一概念应用到大型往复压 缩机上, 作为比较各种流量调节技术经济性的评价 指标.

\section{2 流量调节与脉冲信号处理技术的类比}

由于往复压缩机是间歇吸气、间歇排气的，压缩 机的流量输出可视作周期性的脉冲输出. 我们将压 缩机的流量调节和脉冲信号处理技术相类比.

在电子学和信息处理技术中, 矩形脉冲是经常 遇到的信号. 将单个矩形脉冲的能量 $w$ 定义为幅值 $A$ 和时间宽度 $\tau$ 的乘积, 即 $w=A \tau$. 在时间 $T$ 内将 $N$ 个脉 冲构成的脉冲序列施加到 1 个具有惯性的对象上，各 个脉冲之间的间隔为 $s$, 从能量的角度看, 这等价于 在相同的时间 $T$ 内施加了 1 个与该脉冲序列能量之和 $W$ 相等的直流量 $\bar{A}$ :

$$
W=\sum_{j=1}^{N} w_{j}=\sum_{j=1}^{N} A_{j} \tau_{j} \simeq \int_{0}^{T} \bar{A} \mathrm{~d} t=\bar{A} T,
$$

其中, 脉冲幅值 $A$ 和脉冲群的直流量 $\bar{A}$ 都具有功率 的意义, 而时间 $T$ 满足:

$$
T=\sum_{j=1}^{N}\left(\tau_{j}+s_{j}\right) .
$$

通过改变 $A, \tau, s, N$, 式(4)与(5)提供了多种调节直 流量 $\bar{A}$ 的手段：一种直观而简单的方法是将所有脉 冲的幅值 $A$ 做统一的伸缩, 称为幅度调制 (PAM, pulse amplitude modulation). 当 $\tau, s$ 之和不变而两者 互有消长时，称为脉宽调制(PMW, pulse width modulation). 以上 2 种方式都是脉冲个数不变而调整单 个脉冲的能量, 第 3 种方式是单个脉冲的能量不变而 改变时间 $T$ 内发出脉冲的总个数 $N$, 称为频率调制 (PFM, pulse frequency modulation). 这些方法在电子 技术中都获得了广泛应用.

如前所述, 往复压缩机实际上是按其旋转 1 周为 1 个循环进行周期性工作的. 在压缩机进出口热力参 数等工况条件稳定的情况下, 压缩机末级在每个工 作循环中排出一定质量的气体微团, 我们将其视为 1 个排气质量的脉冲. 质量脉冲具有可与电脉冲相类 比的特征参数：排气行程持续的时间——对应于脉 
冲宽度; 压缩机一个循环的排气质量一一对应于脉 冲幅值; 两个相邻排气行程出现的时间间隔——对 应于脉冲间隔.

利用上述脉冲信号的概念进行分析, 压缩机连 续运转, 实质上是不断的输出一个个的质量脉冲, 由 此形成的脉冲序列施加到压缩机的下游设备——排 气缓冲罐上, 并影响缓冲罐内的压力, 该压力又进一 步影响缓冲罐向下游管道和设备的供气流量. 在一 定压力变化范围内, 可建立缓冲罐的流出质量 $Q_{m o}$ 和 流人质量 $Q_{m i}$ 之间的传递函数为

$$
G(s)=\frac{Q_{m o}(s)}{Q_{m i}(s)}=\frac{1}{T_{c} s+1} \mathrm{e}^{-\tau s},
$$

其中, $T$ 为缓冲罐的暂态过程时间常数, 是其容积和 管道阻力的函数; $\tau$ 为传递滞后, 当仅考虑缓冲罐出 口的流量时, $\tau$ 相对于时间常数 $T$ 可忽略不计. 按式 (6), 缓冲罐是 1 个 1 阶惯性环节, 对压缩机来讲它相 当于 1 个低通滤波器: 它接受压缩机输出的质量脉冲 序列，将其中的高频分量衰减后向下游输出一个“直 流量”——平均的质量流量.

从脉冲信号处理的角度, 压缩机质量流量的定 义式(1)可以改写为如下形式:

$$
Q_{m}=\frac{1}{60 R_{g} T_{s 1}} \cdot n \cdot\left(\lambda_{v 1} \lambda_{p 1} \lambda_{T 1} \lambda_{l 1} V_{h 1} p_{s 1}\right)=k \cdot n \cdot d m, \text { (7) }
$$

其中, $k$ 为不可调系数, $n$ 可理解为单位时间压缩机的 输气次数, $d m$ 的量纲与 $k$ 的量纲结合后具有质量的量 纲, 因此可表征每个工作循环进人气缸的气体质量.

式(7)指明了往复压缩机的流量调节可从两个方 面人手: 或者改变每次进人气缸气体的质量, 或者改 变单位时间内输气的次数. 按照脉冲信号处理的观 点, 它们分别属于调幅和调频两种类型.

\section{2 流量调节技术的发展和现状}

在往复压缩机的各种应用领域中，已经有多种 流量调节方法 ${ }^{[2,3]}$, 根据调节装置作用的部位，可分 为转速调节、管路调节、余隙调节和气阀调节等 4 类. 其中, 转速调节主要包括开/停机控制和连续转 速调节; 管路调节有进气节流、切断进气、排气自由 旁通、排气节流旁通等; 余隙调节是在气缸上连通一 定容积的余隙容积, 它需要改造压缩机气缸的结构; 气阀调节是压开吸气阀使气缸内气体回流到吸气腔. 本文引人脉冲信号概念, 将压缩机流量调节方法按
调幅和调频加以分类, 如表 1 所示

表 1 没有将排气节流旁通方式包含进去. 该方式 是将压缩后多余的气体由旁通阀引出，经节流降压 后送回压缩机进口. 根据定义, 这种方式并不属于严 格意义上的流量调节. 从信号处理的角度看, 该方式 中压缩机相当于 1 个恒流源，节流旁通调节相当于 2 个阻性通路在压缩机出口的并联, 至于流量在两条 通路上如何分配，对压缩机的功率几乎没有影响，始 终保持额定功率，由此造成严重的能量浪费. 但由于 结构简单, 能保证供气连续和压力稳定, 在有些场合 仍有所应用.

表 1 中列举的流量调节方法在动力、制冷等领域 的应用各有千秋. 由于流程的限制和大型压缩机多 级结构的特点, 这些方法是否适用于工艺压缩机还 需要进行针对性的分析. 从实用和安全两个角度考 虑, API 618-2007 标准 ${ }^{[4]}$ 推荐采用排气节流旁通、变 余隙容积和吸气阀卸荷 (或这些方法的组合)作为工 艺压缩机的流量调节方法.

\section{3 调幅型流量调节方法分析}

调幅型流量调节, 就是改变式(7)中 $d m$ 项的值. 升高进气温度可降低温度系数 $\lambda_{T}$, 但会导致功率和 排气温度的升高, 因此不能用于流量调节. 进气节流 改变的是压力系数 $\lambda_{p}$, 但这会进一步改变压缩机级 间压力比的分配，造成活塞力和排气温度升高，这对 大型压缩机是相当有害的, 这种方法目前主要应用 在小型压缩机上. 变余隙调节方法通过增大余隙容 积而减小容积系数 $\lambda_{v}$ 以降低 $d m$ 值, 部分行程压开吸 气阀是通过气体回流而变相的减小泄漏系数 $\lambda_{l}$ 以降 低 $d m$ 值，这两种方法具有一定的应用价值.

变余隙调节方法的原理是：当压缩机气缸的余 隙容积由 $V_{0}$ 增大到 $V_{0}^{\prime}$ 后, 膨胀终了点由 $\mathrm{d}$ 转移到 $\mathrm{d} '$, 指示图变化如图 2 所示. 根据式(2), 当不考虑其他参 数的变化时, 随着余隙容积系数 $\alpha$ 的增大, 压缩机流 量呈线性的减少. 压缩机设计和装配时一般控制

表 1 压缩机流量调节方法的分类

\begin{tabular}{cc}
\hline 调幅 & 调频 \\
\hline 进气节流 & 排气自由旁通 \\
& 切断进气 \\
可变余隙容积调节 & 开/停机控制 \\
& 连续转速调节 \\
部分行程压开吸气阀 & 全行程压开吸气阀 \\
\hline
\end{tabular}




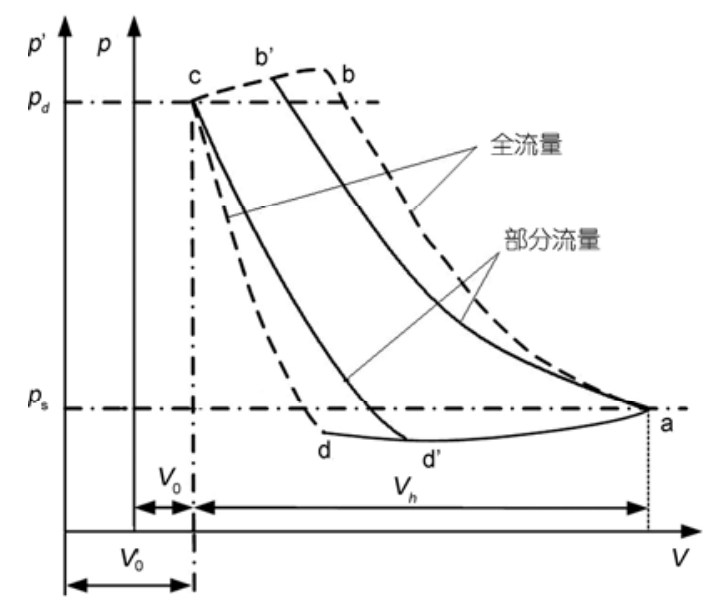

图 2 变余隙调节过程的指示图

$\alpha<0.3$, 可调范围很窄; 通过增加额外的余隙机构, 可以做到 $\alpha>1$. 设膨胀过程指数 $m=1.3$, 压缩机级的 压比 $\varepsilon=2$, 理论上当余隙容积系数 $\alpha>1.42$ 时该级流量 降为零.

对比式(1)和(3)可知, 余隙容积系数对流量和功 率的影响是相同的, 比功率基本保持恒定, 与排气节 流旁通方式相比, 变余隙调节的节能效果非常明显. 美国西南研究院对这类调节方法进行了调研 ${ }^{[5]}$, 认为 它是未来很有潜力的方向之一. 但在调节的幅度较 大时, 会改变压缩机的受力情况, 应注意校核. 此外, 该方式对流量的调节精度比较粗粘.

部分行程压开吸气阀的调节方法, 是在压缩行 程的开始阶段, 伺服机构使吸气阀保持打开, 让气缸 内一部分气体回流到吸气腔; 在特定时刻关闭吸气 阀, 气缸内剩余的气体被封闭、压缩、排出, 从而得 到所需要的气量. 其 $p-V$ 指示图如图 3 所示. 利用该 方法的典型产品如奥地利 Hoerbiger 公司的 HydroCOM 系统 ${ }^{[6,7]}$, Dress-Rand 公司 ISC(Infinite Stepless Controller)系统 ${ }^{[8,9]}$, 国内由浙江大学和镇海炼化联合攻 关研制 DHU 系统经多年改进, 已获得工业实际应 用 ${ }^{[10 \sim 18]}$. 该方法只压缩需要的气量, 比功率接近恒 定, 与排气节流旁通方式相比, 节能效果也很明显, 并可实现无级调节. 但为了达到理想效果, 对执行机 构的性能和可靠性的要求极为苛刻. 若应用于 600 $\mathrm{r} / \mathrm{min}$ 的压缩机上, 伺服机构的响应时间只有几毫秒, 对运动部件抗磨损和抗疲劳的要求也非常高. 随着 大型压缩机向高速发展 ${ }^{[5]}$, 这种调节方法推广的难度 加大.

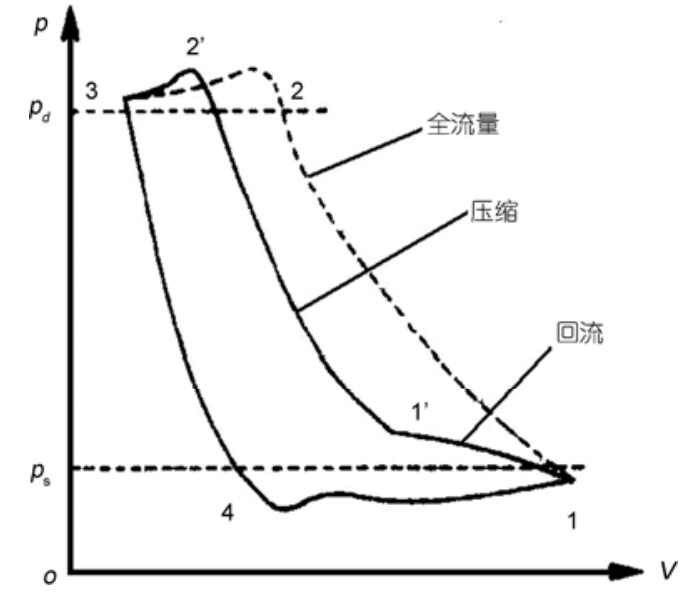

图 3 部分行程压开吸气划

\section{4 调频型流量调节方法分析}

调频型流量调节方法通过改变式(7)中的 $n$ 而实 现. 表 1 中列举的几种调频型调节方法, 只有变转速 调节是连续性调节, 它主要依赖变频器和变频电机 实现, 虽然现有的大容量变频调速技术的调节范围 较窄, 其技术经济性也有待提高, 但这是一个当今世 界范围内非常活跃的研究领域. 其余方法都是间断 性调节, 会造成供气压力的较大波动. 其中, 开/停 机控制不能用于大型压缩机的流量调节. 排气自由 旁通可以用于临时卸载, 不能用于长期的流量调节; 切断进气会改变级间压力比, 对压缩机的安全也是 有害的. 目前适用于大型压缩机的调频型流量调节 方法只有全行程压开吸气阀方法.

\section{1 全行程压开吸气阀}

全行程压开吸气阀是在整个压缩和排气行程中 吸气阀始终被强制压开, 气缸内气体全部回流, 压缩 机不排气, 只消耗很少的能量. 其 $p-V$ 指示图如图 1 中实线所示, 由吸气线 (e-f-a)和回流线 (a-g-e)两条曲 线构成. 依据排气缓冲罐内的压力是否到达上下限 而决定吸气阀被压开或释放. 实现这种操作的伺服 机构是卸荷阀 ${ }^{[19,20]}$. 从脉冲信号处理的角度看, 这种 方法相当于脉冲序列的整体施加或撤消，等同于开 关控制. 这会增加上下游管道内的压力波动. 气量调 节的效果不精确, 只能提供有级调节. 针对卸荷阀的 改进应用, 国内有武汉理工大学 ${ }^{[21,22]}$ 和天华化工机 械及自动化研究设计院 ${ }^{[23]}$ 正从不同的角度分别展开 研究攻关. 


\section{2 占空比调制的流量调节方法}

我们提出一种占空比调制 (duty cycle regulating, DCR) 全行程压开吸气阀的排气量调节方法 ${ }^{[24]}$. 它是 在全行程压开吸气阀调节的基础上, 将压缩机加载 和卸载的开关式控制改进为按脉冲序列的占空比进 行控制.

脉冲序列的占空比定义为各脉冲的持续时间之 和与脉冲序列持续的时间 $T$ 之比，即

$$
R=\frac{1}{T} \sum_{i=1}^{N} \tau_{i}
$$

对于由等宽脉冲构成的序列, 式(8)可简化为 $R=N \tau / T$, 结合式(4)可得到脉冲序列的等效直流量 为 $\bar{A}=R A$. 这说明, 通过改变脉冲序列的占空比即可 获得不同的直流量, 该方法因此被称为占空比调制. 如前所述, 压缩机工作时的输气过程可视为等宽的 排气脉冲序列, 那么从逻辑上进行类比, 占空比调制 的方法亦可应用于压缩机的流量调节. 为了实施这 一方法, 我们提出如下流程:

(1) 以压缩机的 $M$ 个连续工作循环作为 1 个控制 周期;

（2）利用伺服机构压开或释放吸气阀, 使压缩机 交替卸载和加载, 对应的工作循环分别称为卸载循 环和加载循环;

(3) 定义压缩机占空比 $R_{c}$ 为加载循环个数 $N$ 在 控制周期内所占的比例, 即 $R_{c}=N / M$;

（4）若满负荷时压缩机的流量为 $Q$, 则压缩机占 空比为 $R_{c}$ 时的流量为 $\bar{Q}=R_{c} Q$.

上述过程即为占空比调制流量调节方法的基本 原理. 其实质是在式(5)中保持脉冲的参数不变而改 变脉冲出现的间隔 $s$, 从而改变周期 $T$ 内脉冲的个数 $N$, 因此也属于调频型调节方法. 例如，为实现 $70 \%$ 的流量, DCR 模式的流量调节的动作过程如图 4 左侧 一组所示. 图中假定压缩机转动 10 转为一个控制周 期, $M=10$; 令 3 个循环被卸载, 如第 3, 6, 9 个循环, 其进气量为零(图中以空白脉冲表示), 其余 7 个循环 正常加载, 进气量仍为 $100 \%$, 则 $N=7, R_{c}=70 \%$, 整个 周期的平均流量负荷率也为 $70 \%$. 图 4 中右侧一组为 100\%流量, 以示两种情况下的对比. 从图 4 中亦可 看出, DCR 调节时压缩机的比功率保持定值.

DCR 模式有 3 个参数可供调节: 压缩机的占空 比 $R_{c}$ 、控制周期长度 $M$ 和脉冲序列的排列方式, 其

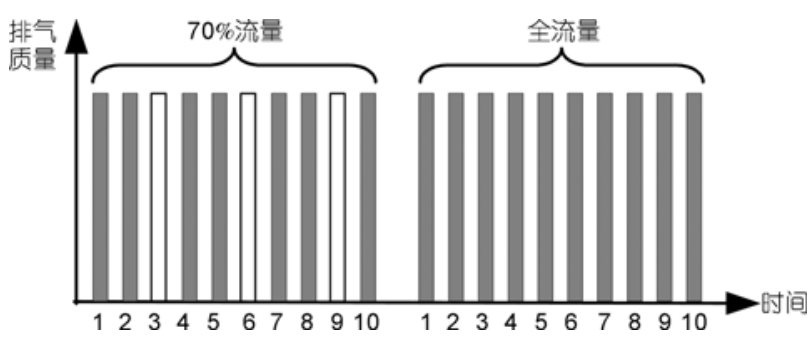

图 4 DCR 模式的操作过程

中, $R_{c}$ 对应于流量的输出百分比, 后两者用以改善调 节效果。

控制周期的长度 $M$ 应限定在合适的范围. 若控 制周期设定的过长，就有可能导致压缩机经历足够 长时间的加载过程使排气缓冲罐内压力超过压力上 限, 或经历足够长时间的卸载过程使排气缓冲罐内 压力低于压力下限, 这样虽然名义上是按照 DCR 模 式的要求在施加主动控制, 但客观上仍然会产生与 开关控制相同的后果. 相反，如果控制周期取得过 短，就限制了流量调节的步进幅度，上例中取 10 个 工作单元为 1 个控制周期, 最多只能产生 10 种占空比, 流量调节的精度以 10\%跃进，蜕化为无级调节. 总之， 控制周期要取得足够长，以保证必要的流量调控精 度; 同时又要取得足够短，以抑制排气压力的波动.

在控制周期长度 $M$ 和占空比 $R$ 确定的情况下, 脉冲序列的不同排列方式即卸载循环出现的位置不 同，会对排气压力有轻微的影响，同时由于存在“呼 吸效应”(见 5.3 节讨论)，对实际的流量产生一定的影 响. 无论调节深度如何, 加载循环和卸载循环在控制 周期内尽量接近均匀相间的排列是较理想的序列构 成方式.

\section{5 占空比调制与其他吸气阀调节方法的对比}

\section{1 调节性能的对比}

HydroCOM 系统、卸荷阀和 DCR 模式都属于吸 气阀调节, 下面依据脉冲信号理论对这 3 种方法的性 能加以对比.

HydroCOM 系统动作过程示于图 5. 仍以输出 $70 \%$ 流量为例, HydroCOM 要求压缩机每个工作循环 中执行机构都必须执行一套完整的动作，压缩机每 个工作循环都有排气，但每个排气脉冲的幅值减少 为 $70 \%$ (图 5 中左侧一组, 以较矮的脉冲高度表示), 


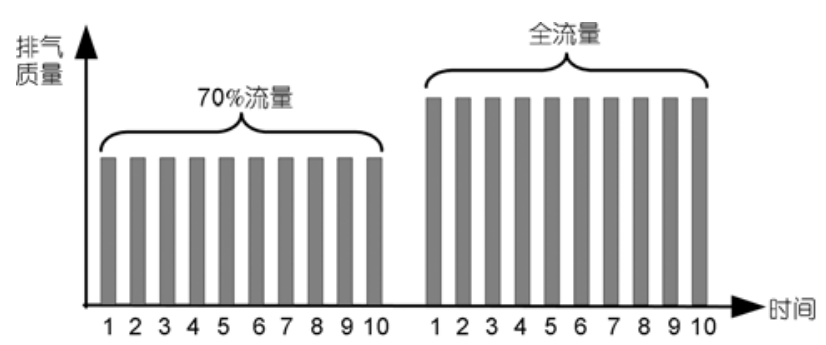

图 5 HydroCOM 的操作过程

平均的排气量就减为 70\%. 图 5 中右侧一组为全流量, 以示对比. 将图 4 与图 5 相对照, 可以看出两者在调 节精度和节能效果上相当, 但 DCR 模式的操作次数 大为减少、控制过程更为简洁, 可靠性和预期寿命比 HydroCOM 系统更高.

DCR 模式与卸荷阀在执行过程上的相同点都是 在卸载阶段每个循环中吸气阀始终保持打开, 区别 在于卸载与加载的转换时机不同. 如图 6 虚线箭头所 示. 卸荷阀工作状态的切换发生在排气压力达到缓 冲压力的上限或下限 (如图 6 中时刻 $t_{1}$ 或 $t_{2}$ ). 按照这 种方式工作, 排气压力的波动是必需的. 而在 DCR 模式下, 工作状态切换是按照预定的时间序列发生, 如图 7 中虚线箭头所示. 按照 DCR 模式工作, 压力波 动在其初始阶段尚未达到显著的幅值即开始反向, 低幅的波动经过后冷却器和排气缓冲罐被进一步衰 减, 因此排气压力波动得以有效抑制. 另一方面, 由 于排气压力更趋稳定, 流量调节的精度也比卸荷阀 方式更加精确. 最重要的是, 占空比调制方法在原理 上使压缩机的比功率接近不变.

由以上对比可以得出结论: 与部分行程压开吸 气阀方法相比, DCR 模式下执行机构动作次数大大 减少, 有利于提高可靠性和延长使用寿命; 与开关控 制的全行程压开吸气阀相比, DCR 模式的优点是卸 载循环均匀分布, 可有效抑制排气压力的波动.

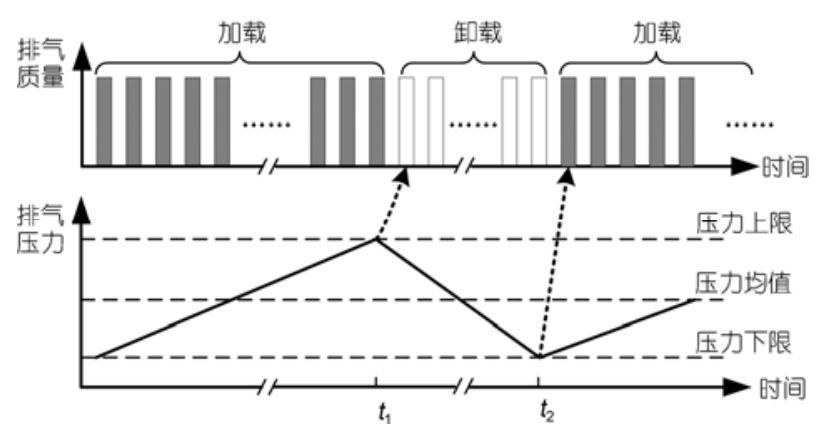

图 6 卸荷阀的状态切换

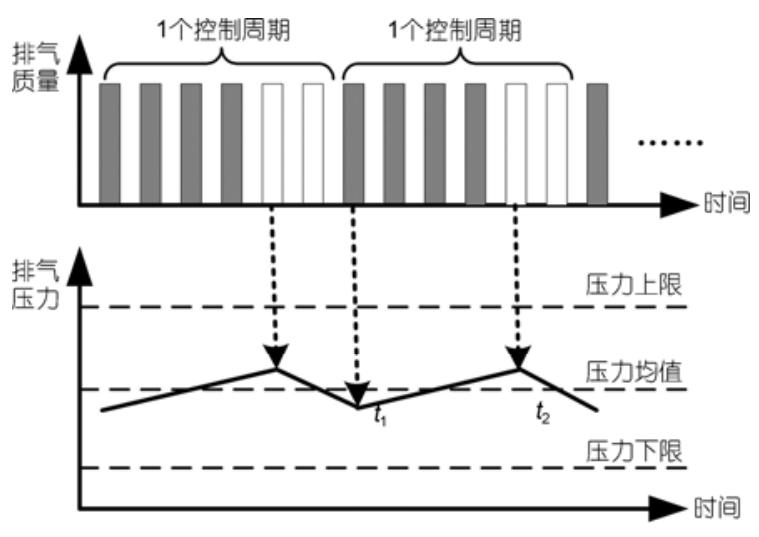

图 7 DCR 模式的状态切换

\section{2 调节方法的实现}

上述 3 种流量调节方法从逻辑上都是对压缩 机的输气脉冲进行操作, 在物理上都需要一套压叉 伸人到阀腔以执行进气阀的压开或释放, 该压叉又 需要一套动力源以推动其运动. 卸荷阀的动力一般 为气动伺服机构, HydroCOM 系统为电-液伺服机 构. DCR 的创新之处在于执行机构的控制逻辑, 即进 气阀压开机构执行动作的时机和每次动作维持的时 间长度. 实现 DCR 方式对压叉的结构没有特殊的要 求; 对执行机构动态特性的要求远低于 HydroCOM 系统, 但比卸荷阀要高. 因此动力源可以像 HydroCOM 系统一样采用液压执行机构; 在进气压 力较低时, 也可以像卸荷阀一样采用气动执行机构. 如果在原有气动执行机构上实施 DCR 方式的新型流 量调节, 要保证其动态性能符合 DCR 的要求; 此外, 控制算法须按照 DCR 模式规定的控制逻辑进行重新 编程, 控制系统的反馈回路与信号连锁等也需进行 重新设计.

另一个需要关注的问题是压缩机在加载和卸载 两种状态的切换瞬间会承受一定的冲击. DCR 模式 下该冲击的强度比卸荷阀弱很多, 但比 HydroCOM 系统略重. 由于各自的工作原理, 卸荷阀和 Hydro COM 系统无法处理这个冲击, 但 DCR 模式下可以通 过合理配置 DCR 在各级和同一级的各个缸之间的动 作次序, 将这一冲击减弱. 为了有效发挥这种新的便 利, 需要详细研究多级压缩机变工况运行特性, 制定 合适的 DCR 调制模式和控制策略.

由于 DCR 模式还处于样机试制和控制程序的开 发阶段, 上述理论模型和类比分析得出的结论尚需 经受最终实验结果的考验. 


\section{3 呼吸效应的影响}

HydroCOM 系统、卸荷阀和 DCR 模式都是以压 开吸气阀为基础的调节方式, 调节的过程都是使气 体在气缸-气阀-吸气腔构成的复杂流通空间内经历 吸人-排出循环, 我们称这种现象为“呼吸效应”. 呼 吸效应的本质是一种非定常流动与复杂换热过程相 耦合的工程热物理问题. 呼吸效应首先会使气缸吸 气温度升高, 进而引起排气温度的升高、功率的增加 和气缸润滑变坏等后果, 且随调节的深度增加而越 发强烈. 同时每个排气脉冲的幅值低于设想的恒定 值, 实际获得的流量有所减少. 因此呼吸效应对压缩 机的运行和流量调节不容忽视. 李黄 ${ }^{[25]}$ 针对 $3 \mathrm{~L}-10 / 8$ 压缩机, 利用集总参数法对 3 种吸气阀调节方法的呼 吸效应进行了初步计算, 呼吸效应的影响定性分析 的结果为:

卸荷阀 > P HydroCOM > DCR.

但目前对呼吸效应的发生机理和影响因素的了 解总体还很肤浅, 在占空比调制方法的实施过程中 尚需深人细致的实验和理论研究.

\section{6 结论}

大型往复压缩机应用广泛、能耗巨大, 在运行中
经常需要进行流量调节, 而能耗与流量紧密相关, 先 进的流量调节技术对于流程行业实现大规模节能减 排具有重要意义. 本文分析了压缩机流量调节方法 的理论基础和现状, 首次引入脉冲信号概念将现有 的各种调节方法分类为调幅和调频两大类型, 重点 介绍了变余隙调节、部分行程压开吸气阀、全行程压 开吸气阀和占空比调制调节方法和系统的原理.

从脉冲信号处理理论出发, 分析了占空比调制 模式、开关式全行程压开吸气阀与部分行程压开吸气 阀等 3 种方法在原理上的异同, 并从系统可靠性、供 气稳定性、气量精确性、节能效果、实现方法等方面 加以对比. 与开关控制的全行程压开吸气阀相比, 占 空比调制模式的供气更稳定, 可有效抑制排气压力 的波动, 调节的精度和节能效果更佳; 与部分行程压 开吸气阀相比, 调节精度和节能效果相当, 但占空比 调制模式下执行机构动作次数大大减少, 有利于提 高可靠性和延长使用寿命.

上述 3 种吸气阀调节方法均存在呼吸效应, 即气 体在流通空间内不断的被吸人和回流. 相对其他 2 种 方式, 在占空比调制模式下呼吸效应的影响较弱. 呼 吸效应对压缩机流量调节的效果和安全运行产生不 利影响, 应在呼吸效应的发生机理和影响因素方面 开展更深人的研究.

\section{参考文献}

1 郁永章. 容积式压缩机技术手册: 动力·化工·制冷. 北京: 机械工业出版社, 2005.44-56

2 任智, 王存智. 往复式压缩机流量调节方式浅析. 石油化工设备技术, 2003, 24: 21-23

3 金江明, 洪伟荣, 梁萌, 等. 往复压缩机气量调节方法的研究进展. 压缩机技术, 2007, 4: 28-32

4 API Standard 618: Reciprocating Compressors for the Petroleum, Chemical and Gas Industry Services. 5th ed. Washington DC: American Petroleum Institute Publishing Services, 2007

5 Danny M D, Klaus B, Ralph E H, et al. Advanced reciprocating compression technology. In: McDonald K, ed. Proceedings of the 2005 GMRC Gas Machinery Conference, Oct 2005, Covington, Kentucky. Dallas: Gas Machinery Research Council Press, 2005. ARCT 1-23

6 Bauer F. Method for Continuous Control of Delivery Rate of Reciprocating Compressors and Device for Carrying Out the Method. US Patent, US5244357-A, 1993-9-14

7 Steinruck P, Ottitsch F, Oberhuber A, et al. Better reciprocating compressor capacity control. Hydrocarb Process, 1997, 76: 79-83

8 McKinney G D, Foreman M L. Active compressor valve control provides maximum unit flexibility. In: Ely C G, ed. Proceedings of the 2001 Gas Machinery Conference, Oct 2001, Austin, Texas. Dallas: Gas Machinery Research Council Press, 2001. 357-362

9 White K H. Infinitely variable capacity control. In: Soedel W, Cohen R, Hamilton J F, et al, eds. Proceedings of the 1972 International Compressor Technology Conference at Purdue, Jul 1972, West Lafayette, Indiana. West Lafayette: Purdue University Press, 1972. 47-51

10 Hong W, Jin J, Wu R, et al. Theoretical analysis and realization of stepless capacity control for reciprocating compressors. Proc Inst Mech Eng Part E-J Process Mech Eng, 2009, 223: 205-213

11 宋健. 活塞式压缩机排气量全量程无级调节系统的开发. 硕士学位论文. 杭州: 浙江大学, 2006

12 孙学亮. 往复压缩机压开吸气阀气量调节的理论与实验研究. 硕士学位论文. 杭州: 浙江大学, 2006

13 张莣. 阀配流式活塞式压缩机变排量控制系统的研究. 硕士学位论文. 杭州: 浙江大学, 2006

14 管宇辉. 活塞式压缩机全量程可调气阀的理论与实验研究. 硕士学位论文. 杭州: 浙江大学, 2005 
15 金江明. 活塞式压缩机排气量无级调节系统关键技术的研究. 博士学位论文. 杭州: 浙江大学, 2010

16 吴荣仁, 刘生礼, 陆君毅. 压缩机吸气阀部分行程顶开时缸内气体压力的计算. 低温工程, 2000, 5: 37-40

17 吴荣仁，管宇辉. 全量程可调气阀的调节机构和最优调节力的分析. 流体机械, 2005, 33: 22-25

18 吴荣仁, 宣海军, 洪伟荣. 部分行程顶开吸气阀调节装置施力压叉的断裂失效分析. 化工机械, 2001, 28: 277-280

19 吴朝华. 带指示器的气动顶开吸气阀调节装置. 压缩机技术, 2005, 6: 29-30

20 梁涌. 往复压缩机气量无级调节系统的原理及应用. 压缩机技术, 2007, 3: 13-17

21 沈顺成, 饶恕. PLC 在往复式压缩机气量调节系统中的应用. 武汉理工大学学报(信息与管理工程版), 2009, 31: 275-277

22 饶恕. 活塞式压缩机气量分时调节系统的研究. 硕士学位论文. 武汉: 武汉理工大学, 2009

23 纪燕飞. 迷宫压缩机气量调节装置的设计与应用. 化工机械, 2008, 35: 4-6

24 顾兆林, 侯雄坡, 李云, 等. 一种往复活塞压缩机排气量无级调节方法. 中国专利. CN 101173658B, 2010-08-25

25 李黄. 一种活塞压缩机气量调节方法的理论研究与实验设计. 硕士学位论文. 西安: 西安交通大学, 2009

- 动 态 $\cdot$

\section{中国-澳大利亚功能纳米材料联合实验室国际合作研究进展}

厦门大学朱贤方教授领导的中国-澳大利亚功能纳 米材料联合实验室，在科技部中澳科技合作特别基金和 国家科技计划国际科技合作与交流专项基金资助下，在 纳米化学生物环境传感器课题研究方面取得了一系列突 破性成果. 课题研究在纳米结构可控制备、纳米结构宏 观有序构筑、纳米结构表面修饰及稳定性、纳米光学传 感模拟及测量等 5 个纳米研究前沿方向均取得了重要突 破. 另外, 围绕课题研究, 联合实验室积极与澳大利亚 国家功能纳米材料研究中心实行频繁的研究资源、设备 和信息共享、研究人员互访交流、研究生联合培养等, 建 立起了互补式且富有成效的合作关系. 先后成功联合申 请到合作研究项目 10 余项, 联合培养研究生 20 余名, 邀请澳大利亚、美国等知名院士、教授、专家来华访问、 讲学、交流、合作 50 多人次. 共计实现了中澳中美研究 人员互访交流、培训、研究生联合培养近 100 人次, 联 合主办高规格功能纳米材料国际学术会议 2 次, 合作申
请专利 7 项, 发表论文 40 余篇, 联合实验室突出的合作 成果及实验室制备出了目前已知世界最好纳米球壳光学 传感关键结构, 被重点推选在 2010 上海世博会澳大利亚 -中国科技周上展示，并被列为中澳科技合作 30 年 30 个 最为成功故事之一. 中国科技部部长万刚和澳大利亚创 新、工业、科学与研究部部长 Senator Kim Carr 专门为 合作成果题了词.

中国-澳大利亚功能纳米材料联合实验室长期联合 中澳双方实验室技术力量(包括 10 余名中国科学院院士 和澳大利亚联邦教授组成的实验室学术委员会), 发挥 中澳双方实验室各自的优势和特长, 开展纳米科学与技 术在生物能源、信息技术、生态环境等领域中前沿战略 性的研究与应用, 推动和促进物理、化学、材料、生物 医学等学科的交叉发展, 为发展我国的纳米科学做出了 重要贡献. 同时在促进亚太地区纳米研究的国际交流与 合作上扮演着重要角色. 